\title{
ORTHOGONAL SYSTEMS OF HYPERSURFACES IN A GENERAL RIEMANN SPACE*
}

BY

\section{LUTHER PFAHLER EISENHART}

1. Introduction. Consider a general Riemann space of $n$ dimensions and write its fundamental quadratic form thus,

$$
d s^{\mathbf{2}}=g_{i j} d x^{i} d x^{j} \quad\left(g_{i j}=g_{j i}\right) . \dagger
$$

If $\alpha_{i j}$ are the components of any other covariant symmetric tensor of the second order, and $\varrho_{h}$ is a root of the equation

$$
\left|\alpha_{r s}+\varrho g_{r s}\right|=0
$$

the functions $\lambda_{h}^{r}(r=1, \ldots, n)$, defined by

$$
\left(\alpha_{r s}+\varrho_{h} g_{r s}\right) \lambda_{h}^{r}=0,
$$

are the contravariant components of a vector in the space.

By hypothesis the form (1) is positive definite and consequently the roots of (2) are real. If all the roots are simple, equations (3) define uniquely $n$ directions at a point. Thus if $\lambda_{h}^{r}$ and $\lambda_{k}^{r}$ are the components of the vectors corresponding to distinct roots $\varrho_{h}$ and $\varrho_{k}$, it follows from (3) that

$$
g_{r s} \lambda_{h}^{r} \lambda_{k}^{s}=0,
$$

that is, the corresponding directions are orthogonal. Moreover, if $\varrho_{h}$ is a multiple root of order $m, \ddagger$ equations (3) admit solutions linearly expressible in terms of $m$ solutions. $\S$ If we take $m$ of these directions which are mutually orthogonal, and proceed in like manner with every multiple root, we have at

* Presented to the Society, April 28, 1923.

† Here $i$ and $j$ are summed from 1 to $n$ in accordance with the convention now generally in use. This convention will be used throughout this paper.

$\ddagger$ We exclude the case where $m=n$, that is, when $a_{r o}+\rho g_{r e}=0$.

$\S$ Weierstrass, Monatsberichte der Akademie zu Berlin, 1858, p. 207. 
each point of space $n$ directions mutually orthogonal. They are the principal directions determined by the tensor $\alpha_{r s}$. These directions determine $n$ congruences of curves in the space, any congruence being defined analytically by

$$
\frac{d x^{1}}{\lambda_{h}^{1}}=\cdots=\frac{d x^{n}}{\lambda_{h}^{n}} .
$$

It is the purpose of this paper to determine conditions necessary and sufficient that the curves of each congruence be orthogonal to a family of hypersurfaces, $\nabla_{n-1}$. In this case, there pass through a curve $C_{h}$, of the congruence defined by (5), $n-1$ hypersurfaces respectively orthogonal to the congruences $C_{k}(k=1, \ldots, n ; k \neq h)$. When these hypersurfaces are taken as parametric, the functions $g_{i j}(i \neq j)$ in (1) are equal to zero, and the space possesses an $n$-uple orthogonal system of hypersurfaces. In this case we say that the $n$-uple of congruences is normal.

When the roots of (2) are simple or double the conditions are readily obtained, but for roots of third and higher order the conditions are quite involved. However, in each case the problem is reduced to an algebraic one.

2. General equations. Without loss of generality, we assume that the functions $\lambda_{h}^{r}$ are chosen so that

$$
g_{r s} \lambda_{h}^{r} \lambda_{h}^{s}=1
$$

As given by (3) these functions are the contravariant components of the direction. Their covariant components are given by

$$
\lambda_{h, r}=g_{r p} \lambda_{h}^{p}
$$

If $\lambda_{h, r / s}$ denote the components of the tensor which is the covariant derivative of $\lambda_{h, r}$ with respect to (1), the functions $\gamma_{h i j}$, defined by

$$
\gamma_{h i j}=\lambda_{i}^{r} \lambda_{j}^{s} \lambda_{h, r / s}
$$

are invariants; they are called rotations by Ricci and Levi-Civita.*

From (8) it follows that

$$
\lambda_{h, r / s}=\sum_{i, j}^{1 \ldots n} \gamma_{h i j} \lambda_{i, r} \lambda_{j, s}
$$

* Mathematische AnnaIen, vol. 54 (1901), p. 148; Wright, Invariants of Quadratic Differential Forms, Cambridge Tract, No.9, p. 68. 
If we denote by $g^{i j}$ the cofactor of $g_{i j}$ in the determinant

$$
g=\left|g_{i j}\right|
$$

divided by $g$, we may replace (7) by

$$
\lambda_{h}^{p}=g^{p r} \lambda_{h, r} .
$$

Taking the covariant derivative of (11) and making use of (9), we have

$$
\lambda_{h / s}^{p}=\sum_{i, j}^{1}{ }^{n} \gamma_{h i j} \lambda_{i}^{p} \lambda_{j, s}
$$

since the covariant derivatives of $g_{i j}$ and $g^{i j}$ are zero. Hence

$$
\gamma_{h i j}=\lambda_{i, p} \lambda_{j}^{s} \lambda_{h i s}^{p}
$$

From (4), (6) and (11) we have

$$
\lambda_{h}^{p} \lambda_{k, p}=\delta_{h k},
$$

where

$$
\delta_{h k}=1 \text { for } h=k \text {, and } 0 \text { for } h \neq k \text {. }
$$

Differentiating (14) with respect to $x^{s}$, multiplying by $\lambda_{l}^{s}$ and summing for $s$, we have, in consequence of (9), (12) and (14),

$$
\gamma_{h k l}+\gamma_{k h l}=0 \quad(h, k, l=1, \ldots, n),
$$

and consequently

$$
\gamma_{h h l}=0 \quad(h, l=1, \ldots, n) .
$$

Ricci* has shown that the conditions of integrability of equations (9) may be written in the form

$$
\gamma_{h i, k l}=B_{q r, s t} \lambda_{h}^{q} \lambda_{i}^{r} \lambda_{k}^{s} \lambda_{l}^{t},
$$

\footnotetext{
* Loc. cit., p. 157; Wright, p. 76.
} 
where $B_{q r, s t}$ are the components of the Riemann tensor, that is

$$
\begin{aligned}
B_{q r, s t} & =g_{r p}\left(\frac{\partial \Gamma_{q s}^{p}}{\partial x^{t}}-\frac{\partial \Gamma_{q t}^{p}}{\partial x^{s}}+\Gamma_{\alpha t}^{p} \Gamma_{q s}^{\alpha}-\Gamma_{a s}^{p} \Gamma_{q t}^{\alpha}\right) \\
\Gamma_{q s}^{p} & =\left\{\begin{array}{c}
q s \\
p
\end{array}\right\}=\frac{1}{2} g^{p a}\left(\frac{\partial g_{q \alpha}}{\partial x^{s}}+\frac{\partial g_{s \alpha}}{\partial x^{q}}-\frac{\partial g_{q s}}{\partial x^{\alpha}}\right)
\end{aligned}
$$

and by definition

(20) $\gamma_{h i, k l}=\frac{\partial \gamma_{h i k}}{\partial s_{l}}-\frac{\partial \gamma_{h i l}}{\partial s_{k}}+\stackrel{\sum}{j}^{n}\left[\gamma_{h i j}\left(\gamma_{j k l}-\gamma_{j l k}\right)+\gamma_{j h l} \gamma_{j i k}-\gamma_{j h k} \gamma_{j i l}\right]$,

it being understood that for any function $f$ the expression $\partial f / \partial s_{l}$ means

$$
\frac{\partial j}{\partial s_{l}}=\frac{\partial f}{\partial x^{n}} \lambda_{l}^{m}
$$

From (18), (19) and (20) it follows that

$$
\gamma_{h i, k l}=-\gamma_{i h, k l}=-\gamma_{h i, l k}=\gamma_{k l, h i}
$$

3. Simple roots. The results of the preceding section hold for any orthogonal $n$-uple. We apply them in this section to the $n$-uple determined by (3). Since (4) is satisfied, whether the functions $\lambda_{h}^{r}$ and $\lambda_{k}^{s}$ correspond to different simple roots of (2), or to the same multiple root if such exist, we have from (3)

$$
\alpha_{r s} \lambda_{h}^{r} \lambda_{k}^{s}=0 \quad(h, k=1, \ldots, n ; h, k \neq)
$$

Also from (3) we have

$$
\alpha_{r s} \lambda_{h}^{r} \lambda_{h}^{s}=-\varrho_{h}
$$

in consequence of (6).

If we differentiate (23) with respect to $x^{t}$, we get, in consequence of (12),

$$
\alpha_{r s / t} \lambda_{h}^{r} \lambda_{k}^{s}+\alpha_{r s} \sum_{i, j}^{1}\left(\lambda_{k}^{s} \gamma_{h i j} \lambda_{i}^{r} \lambda_{j, t}+\lambda_{h}^{r} \gamma_{k i j} \lambda_{i}^{s} \lambda_{j, t}\right)=0
$$

where $\alpha_{r s / t}$ is the covariant derivative of $\alpha_{r s}$ with respect to $x^{t}$. Because of (23), (24) and (16) this is reducible to

$$
\alpha_{r s / t} \lambda_{h}^{r} \lambda_{k}^{s}+\sum_{j}^{1 \ldots n}\left(\varrho_{h}-\varrho_{k}\right) \gamma_{h k j} \lambda_{j, t}=0
$$


Multiplying by $\lambda_{l}^{t}$ and summing for $t$, we get

$$
\alpha_{r s i t} \lambda_{h}^{r} \lambda_{k}^{s} \lambda_{l}^{t}+\left(\varrho_{h}-\varrho_{k}\right) \gamma_{h k l}=0 \quad(h \neq k)
$$

Ricci* has shown that a necessary and sutncient condition that an orthogonal $n$-uple be normal is that

$$
\gamma_{h k l}=0 \quad(h, k, l=1, \ldots, n ; h, k, l \neq) \text {. }
$$

Hence from (27) we have the theorem:

If all the roots of equation (2) are simple, a necessary and sufficient condition that the n-uple defined by (3) be normal is that the functions $\lambda_{h}^{r}$ satisfy the conditions

$$
\alpha_{r s / t} \lambda_{h}^{r} \lambda_{k}^{s} \lambda_{l}^{t}=0 \quad(h, k, l=1, \ldots, n ; h, k, l \neq)
$$

From (20) and (28) it follows that for a normal $n$-uple

$$
\gamma_{h i, k l}=0 \quad(h, i, k, l \neq),
$$

and consequently the functions $\lambda_{h}^{r}$ of a normal $n$-uple satisfy

$$
B_{q r, s t} \lambda_{h}^{q} \lambda_{i}^{r} \lambda_{k}^{s} \lambda_{l}^{t}=0 \quad(h, i, k, l \neq)
$$

4. Double roots. Normal congruences in 3-space. We consider next the case when one of the roots of equation (2) is of order $m$. We denote by $\lambda_{i}^{r}(i=1, \ldots, m)$ the contravariant components of $m$ mutually orthogonal directions corresponding to this root and by $\lambda_{e}^{r}(e=m+1, \ldots, n)$ the components corresponding to the other roots, it being understood that if there are one, or more, other multiple roots, the congruences corresponding to this root be taken orthogonal to one another. Consequently (4) and (6) hold.

The contravariant components $\lambda_{i}^{\prime r}(i=1, \ldots, m)$ of any other orthogonal congruences corresponding to the multiple root of order $m$ are given by

$$
\lambda_{i}^{\prime r}=\sum_{a}^{1 \ldots m} t_{i}^{\alpha} \lambda_{a}^{r} \quad(i=1, \ldots, m ; r=1, \ldots, n)
$$

* Loc. cit., p. 151; Wright, p. 70. 
where the $t$ 's are any functions of the $x$ 's subject to the conditions

$$
\sum_{a}^{1 \ldots m} t_{i}^{a} t_{j}^{a}=\delta_{i j}
$$

the $\delta$ 's being defined by (15). We denote by $\gamma_{\text {hke }}^{\prime}$ the rotations for this set of vectors, and seek under what conditions a set of functions $t$ can be found so that

$$
\gamma_{h i j}^{\prime}=0 \quad(h=1, \ldots, m ; i, j=1, \ldots, n ; h, i, j \neq) .
$$

For $i, j=m+1, \ldots, n(i \neq j)$ we have from (13) and (32)

$$
\gamma_{h i j}^{\prime}=\lambda_{i, p} \lambda_{j}^{s} \dddot{\Sigma}_{a}^{m}\left(t_{h}^{a} \lambda_{\alpha / s}^{p}+\lambda_{a}^{p} \frac{\partial}{\partial x^{s}} t_{h}^{a}\right)=\sum_{\alpha}^{1} \dddot{\Sigma}^{m} t_{h}^{\alpha} \gamma_{\alpha i j}
$$

Hence we must have $\sum_{a}^{1} \dddot{\Sigma}^{m} t_{h}^{a} \gamma_{a i j}=0$. Since these equations must be satisfied by $h=1, \ldots, m$, we must have $\gamma_{h i j}=0$.

For $i=m+1, \ldots, n ; j=1, \ldots, m$ we must have in like manner

$$
\sum_{a, \beta}^{1} t_{h}^{m} t_{j}^{\beta} \gamma_{\alpha i \beta}=0 \quad(h, j=1, \ldots, m, h \neq j ; i=m+1, \ldots, n) .
$$

Holding $j$ fixed and varying $h$, we get in consequence of (33)

$$
\frac{\sum_{\beta}^{1 \ldots m} t_{j}^{\beta} \gamma_{1 i \beta}}{t_{j}^{1}}=\cdots=\frac{\sum_{\beta}^{1 \ldots m} t_{j}^{\beta} \gamma_{m i \beta}}{t_{j}^{m}}=\varrho .
$$

From these equations and the above results we find that we must have

$$
\gamma_{h i j}=0 \quad(h=1, \ldots, m ; i=m+1, \ldots, n ; j=1, \ldots, n ; h, i, j \neq),
$$

and

$$
\gamma_{e \alpha \alpha}=\gamma_{e i i} \quad(e=m+1, \ldots, n ; \alpha, i=1, \ldots, m) .
$$

In consequence of (16) we have from (36) also

$$
\gamma_{h i j}=0 \quad(h=m+1, \ldots, n ; i=1, \ldots, m ; j=1, \ldots, n ; h, i, j \neq) .
$$


From the foregoing discussion it follows conversely that if (36), (37) and (38) are satisfied by any set of mutually orthogonal congruences corresponding to a root of order $m$, they are satisfied by every such set. From (27) it follows that (37) is equivalent to

$$
\alpha_{r s \mid t} \lambda_{e}^{r}\left(\lambda_{\alpha}^{s} \lambda_{\alpha}^{t}-\lambda_{i}^{s} \lambda_{i}^{t}\right)=0 \quad(e=m+1, \ldots, n ; \alpha, i=1, \ldots, m) .
$$

Consider next the case where (34) are satisfied when $h, i=1, \ldots, m$; $j=m+1, \ldots, n ; i \neq j$. Now

$$
\gamma_{h i j}^{\prime}=\sum_{a, \beta}^{1} t_{i}^{\beta}\left[t_{h}^{a} \gamma_{a \beta j}+\frac{\partial}{\partial s_{j}}\left(t_{h}^{\beta}\right)\right] .
$$

In consequence of (16) and (33) we have identically

$$
\sum_{a, \beta}^{1 \ldots m} t_{h}^{\beta}\left[t_{h}^{\alpha} \gamma_{\alpha \beta j}+\frac{\partial}{\partial s_{j}}\left(t_{h}^{\beta}\right)\right]=0
$$

From this equation and those obtained by equating to zero the right-hand member of the above equation, we have

$$
\frac{\partial}{\partial s_{j}}\left(t_{h}^{\beta}\right)=\sum_{a}^{1 \ldots m} t_{h}^{\alpha} \gamma_{\beta a j} \quad(\beta, h=1, \ldots, m ; j=m+1, \ldots, n) .
$$

Suppose that the given space is of the third order; that equation (2) admits a double root; that equations (29) and (39) are satisfied and that $\lambda_{3}^{r}(r=1,2,3)$ are the components of the vector corresponding to the simple root of (2). By a transformation of coordinates we can take the surfaces orthogonal to this congruence for the surfaces $x_{3}=$ const. Then $\lambda_{8}^{1}=\lambda_{8}^{2}=0, \lambda_{8}^{8}=1 / \sqrt{g_{88}}$. If we put

$$
t_{1}^{1}=\cos \theta, \quad t_{1}^{2}=\sin \theta, \quad t_{2}^{1}=-\sin \theta, \quad t_{2}^{2}=\cos \theta,
$$

equations (33) are satisfied, and in place of (40) we have the single equation

$$
\frac{\partial \theta}{\partial x^{3}}=\sqrt{g_{33}} \gamma_{213}
$$


for the determination of $\theta$, and consequently of the two normal congruences corresponding to the double root. Hence:

$A$ necessary and sufficient condition that the equations (3) for a 3-space, for which (2) admits a double root, lead to a triple of normal congruences is that equations (29) and (39) be satisfied by the direction corresponding to the simple root and by any set of mutually orthogonal directions corresponding to the double root.

If the space is of higher order than the third, the conditions of integrability of (40) must be considered. These conditions are expressible in the form*

$$
\left(\frac{\partial}{\partial s_{k}} \frac{\partial}{\partial s_{j}}-\frac{\partial}{\partial s_{j}} \frac{\partial}{\partial s_{k}}\right) f+\sum_{r}^{1} \dddot{\Sigma}^{n}\left(\gamma_{r j k}-\gamma_{r k j}\right) \frac{\partial f}{\partial s_{r}}=0
$$

Applying this condition to equations (40), we obtain, in consequence of (36), (38) and (20),

$$
\sum_{a}^{1}{ }^{m} t_{h}^{a} \gamma_{\beta a, j k}=0
$$

Since equations of this form must be hold for $h=1, \ldots, m$, we must have

$$
\gamma_{\alpha \beta, j k}=0 \quad(\alpha, \beta=1, \ldots, m ; j, k=m+1, \ldots, n) .
$$

We have seen in (30) that this condition must be satisfied by the functions $\gamma_{h i j}^{\prime}$ of a normal congruence. From (32) we have

$$
\gamma_{\alpha \beta, j k}^{\prime}=B_{q r, s t} \lambda_{a}^{\prime q} \lambda_{\beta}^{\prime r} \lambda_{j}^{s} \lambda_{k}^{t}=\sum_{a, b}^{1 \ldots m} \gamma_{a b, j k} t_{\alpha}^{a} t_{\beta}^{b}
$$

Hence if (43) is satisfied by any set of congruences corresponding to a multiple root, it is satisfied by every such set.

The conditions (36), (37), (38) and (40) are the only ones applying to a double root of equation (2). Hence:

If the roots of equation (2) are simple and double at most, a necessary and sufficient condition that there exist a normal n-uple whose components satisfy (3)

* Ricci and Levi-Civita, p. 150; Wright, p. 69. 
is that any orthogonal $n$-uple satisfying (3) shall satisfy (29), in which $h$ and $k$ do not refer to the same root of (2), (39), and also the equations

$$
B_{q r, s t} \lambda_{\alpha}^{q} \lambda_{\beta}^{r} \lambda_{j}^{s} \lambda_{k}^{t}=0 \quad(\alpha, \beta, j, k \neq),
$$

in which $\alpha$ and $\beta$ refer to the same double root, and $j$ and $k$ to any other root or roots.

5. Multiple roots of third and higher orders. If $m>2$, we must consider also the case of equations (34) for $h, i, j=1, \ldots, m ; h, i, j \neq$. Now we must have

$$
\sum_{\alpha, \beta, \sigma}^{1 \ldots m} t_{i}^{\beta} t_{j}^{\alpha}\left[t_{h}^{\sigma} \gamma_{\sigma \beta \alpha}+\frac{\partial}{\partial s_{\alpha}} t_{h}^{\beta}\right]=0 \quad(h, i, j=1, \ldots, m ; h, i, j \neq) .
$$

Since this equation is satisfied identically for $i=h$, equations (46) hold for $i=1, \ldots, m$, but $i \neq j$, and consequently (46) may be replaced by

$$
\sum_{a, \sigma}^{1 \ldots m} t_{j}^{a}\left[t_{h}^{\sigma} \gamma_{\sigma \beta a}+\frac{\partial}{\partial s_{\alpha}} t_{h}^{\beta}-A_{h} \delta_{a \beta}\right]=0
$$

where $A_{h}$ is to be determined. Since this equation must be satisfied by all values of $j$ except $h$, it may be replaced by

$$
\frac{\partial}{\partial s_{\alpha}} t_{h}^{\beta}+\sum_{\sigma}^{1 \ldots m} t_{h}^{\sigma} \gamma_{\sigma \beta \alpha}-A_{h} \delta_{a \beta}=B_{\beta} t_{h}^{\alpha}
$$

where $B_{\beta}$ is to be determined. Multiplying this equation by $t_{h}^{\beta}$ and summing for $\beta$, we find that $A_{h}=-\sum_{\beta}^{1} B_{\beta} t_{h}^{\beta}$, and the above equation becomes

$$
\frac{\partial}{\partial s_{\alpha}} t_{h}^{\beta}=\sum_{\sigma}^{1 \ldots m} t_{h}^{\sigma}\left(\gamma_{\beta \sigma \alpha}-\delta_{\alpha \beta} B_{\sigma}+B_{\beta} \delta_{\sigma \alpha}\right) \quad(\alpha, \beta, h=1, \ldots, m) .
$$

We have now to consider the consistency of these equations and (40). However, both sets of equations may be written

$$
\frac{\partial}{\partial s_{\alpha}}\left(t_{h}^{\beta}\right)=\sum_{\sigma}^{1} \sum_{h}^{m} t_{h}^{\sigma}\left(\gamma_{\beta \sigma \alpha}-\delta_{\alpha \beta} B_{\sigma}+B_{\beta} \delta_{\sigma \alpha}\right) \quad\left(\begin{array}{c}
a=1, \ldots, n ; \\
\beta, h=1, \ldots, m
\end{array}\right) .
$$


When we apply conditions of integrability of the form (41) to these equations, we get equations of the form $\sum_{\sigma}^{1 \ldots m} t_{h}^{\sigma} A_{\sigma \beta \alpha e}=0$, where $A_{\sigma \beta a e}$ is the left-hand member of (48). Since equations of this form hold for $h=1, \ldots, m$ it follows that we must have

$$
\begin{gathered}
\gamma_{\beta \sigma, \alpha e}+\sum_{k}^{m+1 \ldots n}\left(\gamma_{\beta k e} \gamma_{k \sigma \alpha}-\gamma_{\beta k \alpha} \gamma_{k \sigma e}\right)+\left(\delta_{e \beta} \delta_{\sigma \alpha}-\delta_{\alpha \beta} \delta_{\sigma e}\right) \sum_{i}^{1} B_{i}^{2} \\
+\delta_{\sigma \alpha}\left[\frac{\partial B_{\beta}}{\partial s_{e}}+\sum_{i}^{1 \ldots m} B_{i}\left(\gamma_{i \beta e}-\delta_{i e} B_{\beta}\right)\right] \\
-\delta_{\beta \alpha}\left[\frac{\partial B_{\sigma}}{\partial s_{e}}+\sum_{i}^{1} \sum_{i}^{m}\left(\gamma_{i \sigma e}-\delta_{i e} B_{\sigma}\right)\right] \\
-\delta_{\sigma e}\left[\frac{\partial B_{\beta}}{\partial s_{\alpha}}+\sum_{i}^{1 \ldots m} B_{i}\left(\gamma_{i \beta \alpha}-\delta_{i \alpha} B_{\beta}\right)\right] \\
+\delta_{\beta e}\left[\frac{\partial B_{\sigma}}{\partial s_{\alpha}}+\sum_{i}^{1 \ldots m} B_{i}\left(\gamma_{i \sigma \alpha}-\delta_{i \alpha} B_{\sigma}\right)\right]=0
\end{gathered}
$$

for $\beta, \sigma=1, \ldots, m ; \alpha, e=1, \ldots, n ; \alpha \neq e$.

For $\beta, \sigma, \alpha, e$ all different, equation (48) becomes, in consequence of (36) and (38),

$$
\gamma_{\beta \sigma, \alpha e}=0 \quad(\beta, \sigma=1, \ldots, m ; \alpha, e=1, \ldots, n ; \beta, \sigma, \alpha, e \neq) .
$$

If we take $\sigma=\alpha ; \beta, \alpha, e \neq$ or $\beta=\alpha ; \sigma, \alpha, e \neq$, we obtain from (48) equations of the form

$$
\begin{aligned}
\frac{\partial B_{\beta}}{\partial s_{e}}=\sum_{i}^{1 \ldots m} B_{i}\left(\gamma_{\beta i e}+\delta_{i e} B_{\beta}\right)-\left(\gamma_{\beta \alpha, \alpha e}+\sum_{k}^{m+1 \ldots n} \delta_{k e} \gamma_{\beta k k} \gamma_{k \alpha a \alpha}\right) \\
\\
\left(\begin{array}{l}
\beta, i, a=1, \ldots, m ; \\
e=1, \ldots, n ; \beta \neq e
\end{array}\right) .
\end{aligned}
$$

If we take $\sigma=\beta$, equation (48) vanishes identically.

If we take $\sigma=\alpha, \beta=e$, or $\sigma=e, \beta=\alpha$, we get an equation of the form

$$
\frac{\partial B_{e}}{\partial s_{e}}+\frac{\partial B_{\alpha}}{\partial s_{\alpha}}-B_{e}^{2}-B_{\alpha}^{2}+\sum_{i}^{1 \ldots m} B_{i}\left[\gamma_{i e e}+\gamma_{i \alpha \alpha}+B_{i}\right]+\gamma_{e \alpha, \alpha e}
$$

$$
-\sum_{k}^{m+1 \ldots n} \gamma_{k e e} \gamma_{k \alpha \alpha}=0 \quad(\alpha, e=1, \ldots, m ; \alpha, e \neq) .
$$


From (50) and (37) it follows that we must have

$$
\gamma_{\beta i, i e}=\gamma_{\beta \alpha, \lambda e} \quad(\alpha, \beta, i=1, \ldots, m ; e=1, \ldots, n ; \alpha, \beta, i, e \ddagger) .
$$

If this equation is written in the form $\gamma_{e i, i \beta}=\gamma_{e a, a \beta}$ it follows that from (20), (36), (37) and (38) this equation is satisfied identically for $e=m+1, \ldots, n$. In consequence of (37) and (52) we put for the sake of brevity

$$
\begin{aligned}
& \gamma_{\beta e}=\gamma_{\beta i, i e}+\gamma_{\beta e e} \gamma_{e i i} \\
& \gamma_{\beta a}=\gamma_{\beta i, i \alpha}
\end{aligned} \quad(\beta, i, \alpha=1, \ldots, m ; e=m+1, \ldots, n ; \beta, i, \alpha \neq)
$$

and write equations $(50)$ in the form

(54) $\frac{\partial B_{\beta}}{\partial s_{e}}=\sum_{i}^{1} \varlimsup^{m} B_{i}\left(\gamma_{\beta i e}+\delta_{i e} B_{\beta}\right)-\gamma_{\beta e}(\beta, i=1, \ldots, m ; e=1, \ldots, n ; \beta, e \neq)$.

When we apply to (54) conditions of integrability of the form (41), we obtain

$$
\begin{gathered}
\sum_{i}^{1 \ldots m} B_{i}\left[\gamma_{\beta i, a e}+\delta_{i e} \gamma_{\beta a}-\delta_{i a} \gamma_{\beta e}+\gamma_{\beta a e}\left(\gamma_{i \beta \beta}-\gamma_{i a \alpha}\right)-\gamma_{\beta e a}\left(\gamma_{i \beta \beta}-\gamma_{i e e}\right)\right. \\
\left.+\delta_{i a} B_{a} \gamma_{\beta a e}-\delta_{i e} B_{e} \gamma_{\beta e a}+\sum_{k \neq a}^{m+1 \ldots n} \gamma_{\beta k e} \gamma_{k i a}-\sum_{k \neq e}^{m+1 \ldots n} \gamma_{\beta k a} \gamma_{k i e}\right] \\
\left.(55)+\sum_{i}\left[\frac{\partial B_{i}}{\partial s_{i}}\left(\delta_{i e} \gamma_{\beta i a}-\delta_{i a} \gamma_{\beta i e}\right)+B_{i} B_{\beta}\left(\sum_{j}^{1} \sum_{j a}^{m} \delta_{j i e}-\delta_{j e} \gamma_{j i a}\right)+\gamma_{i a e}-\gamma_{i e a}\right)\right] \\
+\left(\gamma_{\beta a e}-\gamma_{\beta e a}\right)\left(\frac{\partial B_{\beta}}{\partial s_{\beta}}-B_{\beta}^{2}\right)+B_{\beta}\left(\sum_{i \neq a}^{1 \ldots m} \delta_{i e} \gamma_{i a}-\sum_{i \neq e}^{1 \ldots m} \delta_{i a} \gamma_{i e}\right) \\
+\frac{\partial \gamma_{\beta e}}{\partial s_{a}}-\frac{\partial \gamma_{\beta a}}{\partial s_{e}}+\sum_{j \neq a}^{1 \ldots m} \gamma_{\beta j e} \gamma_{j a}-\sum_{j \neq e}^{1 \ldots m} \gamma_{\beta j a} \gamma_{j e}+\sum_{k \neq \beta}^{1 \ldots n} \gamma_{\beta k}\left(\gamma_{k e a}-\gamma_{k a e}\right)=0 .
\end{gathered}
$$


For $\alpha, e=m+1, \ldots, n$ we have from (55), in consequence of (36), (38) and (49),

(56) $\frac{\partial \gamma_{\beta e}}{\partial s_{\alpha}}-\frac{\partial \gamma_{\beta a}}{\partial s_{e}}+\sum_{j}^{1 \ldots m} \gamma_{\beta j e} \gamma_{j a}-\sum_{j}^{1 \ldots m} \gamma_{\beta j a} \gamma_{j e}+\sum_{k}^{m+1 \ldots n} \gamma_{\beta k}\left(\gamma_{k e a}-\gamma_{k a e}\right)=0$.

For $\alpha=1, \ldots, m ; e=m+1, \ldots, n$ we have, in consequence of (36), (37), (39) and (53),

$$
\begin{gathered}
\frac{\partial \gamma_{\beta e}}{\partial s_{\alpha}}-\frac{\partial \gamma_{\beta \alpha}}{\partial s_{e}}+\sum_{j \neq \alpha}^{1 \ldots m} \gamma_{\beta j e} \gamma_{j \alpha}-\sum_{j}^{1 \ldots m} \gamma_{\beta j \alpha} \gamma_{j e}+\sum_{k \neq \beta}^{1 \ldots n} \gamma_{\beta k}\left(\gamma_{k e \alpha}-\gamma_{k a e}\right) \\
+\gamma_{\beta \alpha e}\left[\frac{\partial B_{\beta}}{\partial s_{\beta}}-B_{\beta}^{2}-\frac{\partial B_{a}}{\partial s_{\alpha}}+B_{\alpha}^{2}+\sum_{i}^{1} B_{i}^{m}\left(\gamma_{i \beta \beta}-\gamma_{i \alpha \alpha}\right)\right]-B_{\beta} \gamma_{a e}+B_{\alpha} B_{\beta} \gamma_{e \alpha \alpha}=0,
\end{gathered}
$$

By means of (51) this is reducible to

$$
\frac{\partial \gamma_{\beta e}}{\partial s_{\alpha}}-\frac{\partial \gamma_{\beta a}}{\partial s_{e}}+\sum_{j \neq a}^{1 \ldots m} \gamma_{\beta j e} \gamma_{j a}-\sum_{j}^{1 \ldots m} \gamma_{g j a} \gamma_{j e}+\sum_{k \neq \beta}^{1 \ldots n} \gamma_{\beta k}\left(\gamma_{k e a}-\gamma_{k a e}\right)
$$

$$
+\gamma_{\beta a e}\left(\gamma_{i \alpha, \alpha i}-\gamma_{i \beta, \beta i}\right)-B_{\beta} \gamma_{a e}+B_{\beta} B_{a} \gamma_{e a \alpha}=0
$$

In like manner for $\alpha, e=1, \ldots, m$, we have

$$
\frac{\partial \gamma_{\beta e}}{\partial s_{\alpha}}-\frac{\partial \gamma_{\beta a}}{\partial s_{e}}+\sum_{j \neq \alpha}^{1 \ldots m} \gamma_{\beta j e} \gamma_{j a}-\sum_{j \neq e}^{1 \ldots m} \gamma_{\beta j a} \gamma_{j e}+\sum_{k \neq \beta}^{1 \ldots m} \gamma_{\beta k}\left(\gamma_{k e a}-\gamma_{k a e}\right)
$$

$$
+\gamma_{\beta a e}\left(\gamma_{e a, \alpha e}-\gamma_{e \beta, \beta e}\right)-\gamma_{\beta e \alpha}\left(\gamma_{\alpha e, e a}-\gamma_{\alpha \beta, \beta a}\right)=0 .
$$

6. Reduced form of conditions (56), (57) and (58). From (18) we have

$$
\gamma_{\beta i, i e}=B_{q r, s t} \lambda_{\beta}^{q} \lambda_{i}^{r} \lambda_{i}^{s} \lambda_{e}^{t}
$$


and by differentiation we have for $\alpha$ different from $\beta, i$ and $e$

$$
\frac{\partial}{\partial s_{\alpha}} \gamma_{\beta i, i e}=B_{q r, s t p} \lambda_{\beta}^{q} \lambda_{i}^{r} \lambda_{i}^{s} \lambda_{e}^{t} \lambda_{a}^{p}+\sum_{j}^{1} \dddot{\sum}^{n}\left[\gamma_{j i, i e} \gamma_{\beta j \alpha}+\gamma_{\beta j, i e} \gamma_{i j \alpha}\right.
$$

$$
\left.+\gamma_{\beta i, j e} \gamma_{i j \alpha}+\gamma_{\beta i, i j} \gamma_{e j \alpha}\right] \quad(\beta, i=1 \ldots \underset{\beta, i, \alpha, e \neq}{m ; \alpha, e=1, \ldots, n ;}) .
$$

Bianchi has established the following identity:*

$$
B_{q r, s t p}+B_{q r, t p s}+B_{q r, p s t}=0 \text {. }
$$

Making use of (61) and the well known identities

$$
B_{q r, s t}=-B_{q r, t s}=-B_{r q, s t}
$$

we obtain

$$
\begin{aligned}
\frac{\partial}{\partial s_{\alpha}} \gamma_{\beta i, i e}- & \frac{\partial}{\partial s_{e}} \gamma_{\beta i, i a}=B_{q r, p t s} \lambda_{\beta}^{q} \lambda_{i}^{r} \lambda_{a}^{p} \lambda_{e}^{t} \lambda_{i}^{s} \\
& +\sum_{j}^{1 \dddot{n}^{n}}\left[\gamma_{j i, i e} \gamma_{\beta j \alpha}+\left(\gamma_{\beta j, i e}+\gamma_{\beta i, j e}\right) \gamma_{i j a}-\gamma_{j i, i a} \gamma_{\beta j e}\right. \\
& \left.-\left(\gamma_{\beta j, i a}+\gamma_{\beta i, j a}\right) \gamma_{i j e}+\left(\gamma_{j a e}-\gamma_{j e a}\right) \gamma_{\beta i, i j}\right]
\end{aligned}
$$

Since $\beta, i, \alpha, e$ are different, we have from (49)

$$
\gamma_{\beta i, a e}=B_{q r, p t} \lambda_{\beta}^{q} \lambda_{i}^{v} \lambda_{\alpha}^{p} \lambda_{e}^{t}=0
$$

Differentiate with respect to $x^{s}$, multiply by $\lambda_{i}^{s}$ and sum for $s$; we get

$$
B_{q r, p t s} \lambda_{\beta}^{q} \dot{\lambda}_{i}^{r} \lambda_{\alpha}^{p} \hat{\lambda}_{e}^{t} \hat{\lambda}_{i}^{s}+\sum_{j}^{1 \ldots{ }^{n}}\left[\gamma_{j i, \alpha e} \gamma_{\beta j i}+\gamma_{\rho j, a e} \gamma_{i j i}+\gamma_{\beta i, j e} \gamma_{\alpha j i}+\gamma_{\rho i, a j} \gamma_{e j i}\right]=0 .
$$

* Lezioni, vol. 1, p. 351. 
Hence in consequence of (49) we obtain

$$
\begin{aligned}
& \frac{\partial}{\partial s_{\alpha}} \gamma_{\beta i, i e}-\frac{\partial}{\partial s_{e}} \gamma_{\beta i, i \alpha}=\gamma_{\beta a i}\left(\gamma_{i \alpha, \alpha e}-\gamma_{i \beta, \beta e}\right)+\gamma_{\beta e i}\left(\gamma_{i \beta, \beta \alpha}-\gamma_{i e, e \alpha}\right) \\
& +\gamma_{\alpha i i}\left(\gamma_{\beta a, \alpha e}-\gamma_{\beta i, i e}\right)+\gamma_{e i i}\left(\gamma_{\beta i, i \alpha}-\gamma_{\beta e, e a}\right)+\gamma_{e i a} \gamma_{\beta e, e i} \\
& +\sum_{j \neq a}^{1 \ldots m} \gamma_{j i, i e} \gamma_{\beta j a}+\gamma_{i \beta, \beta e} \gamma_{\beta i \alpha}+\gamma_{\alpha i, i e}\left(\gamma_{\beta a \alpha}-\gamma_{\beta e e}\right)-\gamma_{\alpha i e} \gamma_{\beta \alpha, a i} \\
& -\sum_{j \neq e}^{1 \ldots j^{m}} \gamma_{j i, i \alpha} \gamma_{\beta j e}-\gamma_{i \beta, \beta a} \gamma_{\beta i e}+\sum_{j}^{1 \ldots n}\left(\gamma_{j a e}-\gamma_{j e \alpha}\right) \gamma_{\beta i, i j} \\
& +\sum_{k \neq \alpha, e}^{m+1 \ldots n} \gamma_{\beta k, \alpha e} \gamma_{k i i}+\gamma_{\beta a, i e} \gamma_{i a \alpha}-\gamma_{\beta e, i a} \gamma_{i e e} .
\end{aligned}
$$

For $\alpha, e=m+1, \ldots, n, \alpha \neq e$, and $\beta, i=1, \ldots, m, \beta \neq i$, we have from (20), in consequence of (36), (37) and (38),

$$
\begin{aligned}
& \frac{\partial}{\partial s_{\alpha}} \gamma_{\beta e e}=\gamma_{\beta e, e \alpha}+\sum_{j}^{1 \ldots m} \gamma_{\beta j a} \gamma_{j e e}+\gamma_{\alpha e e}\left(\gamma_{\beta \alpha a}-\gamma_{\beta e e}\right), \\
& \frac{\partial}{\partial s_{\alpha}} \gamma_{e i i}=\gamma_{e i, i \alpha}-\gamma_{e i i} \gamma_{\alpha i i}+\gamma_{a e i}\left(\gamma_{i e e}-\gamma_{i a \alpha}\right)-\sum_{k}^{m+1 \ldots n} \gamma_{k e \alpha} \gamma_{k i i}, \\
& \gamma_{\beta a, i e}=\gamma_{\alpha e i}\left(\gamma_{\beta e e}-\gamma_{\beta a \alpha}\right), \\
& \gamma_{\beta k, a e}=\gamma_{k a e}\left(\gamma_{\beta k k}-\gamma_{\beta a \alpha}\right)-\gamma_{k e a}\left(\gamma_{\beta k k}-\gamma_{\beta e e}\right) \\
&(k=m+1, \ldots, n ; \alpha, e, k \neq) .
\end{aligned}
$$

By means of these results, equations (64) and (53), defining the function $\gamma_{\beta e}$, we find that (56) is reducible to

$$
\gamma_{e i i} \gamma_{\beta a}-\gamma_{a i i} \gamma_{\beta e}=0 \quad(\beta, i=1, \ldots, m ; \alpha, e=m+1, \ldots, n)
$$

For $\alpha, \boldsymbol{\beta}, i=1, \ldots, m ; e=m+1, \ldots, n$ we have from (20) in consequence of (36), (37) and (38), 


$$
\frac{\partial \gamma_{\beta e e}}{\partial s_{\alpha}}=\gamma_{\beta e, e \alpha}-\gamma_{\beta e e} \gamma_{\alpha e e}-\sum_{j}^{1 \ldots m} \gamma_{j \beta \alpha} \gamma_{j e e},
$$

$$
\begin{aligned}
& \frac{\partial \gamma_{e i i}}{\partial s_{\alpha}}=\gamma_{c i, i a}-\sum_{k}^{m+1 \ldots n} \gamma_{k e \alpha} \gamma_{k i i}, \\
& \gamma_{\beta k, \alpha e}=\gamma_{k e \alpha}\left(\gamma_{\beta e e}-\gamma_{\beta k k}\right) \quad(k=m+1, \ldots, n ; k \neq e) .
\end{aligned}
$$

By means of these results, equations (64) and (53), we find that (57) is reducible to

$$
\gamma_{e i i} \gamma_{\beta \alpha}-B_{\beta} \gamma_{\alpha e}+B_{\beta} B_{a} \gamma_{e i i}=0 \quad\left(\begin{array}{c}
\alpha, \beta, i=1, \ldots, m ; \\
e=m+1, \ldots, n
\end{array}\right)
$$

For $\boldsymbol{\alpha}, \boldsymbol{\beta}, e=1, \ldots, m ; \boldsymbol{\alpha}, \boldsymbol{\beta}, e \neq$ we have

$$
\frac{\partial \gamma_{\beta e}}{\partial s_{\alpha}}=\frac{\partial \gamma_{\beta \alpha, \alpha e}}{\partial s_{\alpha}}=B_{q r, s t p} \lambda_{\beta}^{q} \lambda_{\alpha}^{r} \lambda_{\alpha}^{s} \lambda_{e}^{t} \lambda_{\alpha}^{p}+\sum_{j \neq e}^{1 \ldots m} \gamma_{j e} \gamma_{\beta j \alpha}
$$

$$
+\sum_{j \neq \beta}^{1 \ldots m} \gamma_{\beta j} \gamma_{e j,}+\gamma_{e \alpha, \sigma e} \gamma_{\beta e \alpha}+\gamma_{\beta \alpha, \alpha \beta} \gamma_{e \beta x} .
$$

Substituting in (58), we get

$$
B_{q r, s t p}\left(\lambda_{\beta}^{q} \lambda_{\alpha}^{r} \lambda_{\alpha}^{s} \lambda_{e}^{t} \lambda_{\alpha}^{p}-\lambda_{\beta}^{q} \lambda_{e}^{r} \lambda_{e}^{s} \lambda_{a}^{t} \lambda_{e}^{p}\right)=0 \quad\left(\begin{array}{c}
\beta, a, e=1, \ldots, m, \\
\beta, \alpha, e \neq
\end{array}\right) .
$$

7. Consistency of equations (50) and (5I). If we take the three equations of the form (51) involving three distinct indices $\alpha, \beta$ and $i$, we obtain

$$
\begin{aligned}
\frac{\partial B_{\beta}}{\partial s_{\beta}}=B_{\beta}^{2}-\sum_{j}^{1}\left(B_{j} \gamma_{j \beta \beta}\right. & \left.+\frac{1}{2} B_{j}^{2}\right) \\
& +\frac{1}{2}\left(\gamma_{i \alpha, \alpha i}-\gamma_{i \beta, \beta i}-\gamma_{\alpha \beta, \beta \alpha}\right)+\frac{1}{2} \sum_{k}^{m+1 \ldots n} \gamma_{k i i}^{2} .
\end{aligned}
$$

Expressing the consistency of this equation and (50) for $e=m+1, \ldots, n$ by means of equations of the form (41), we reduce the resulting equations to the form 


$$
\begin{gathered}
\frac{1}{2} \frac{\partial}{\partial s_{e}}\left(\gamma_{i a, a i}-\gamma_{i \beta, \beta i}-\gamma_{a \beta, \beta a}\right)+\frac{\partial \gamma_{\beta e}}{\partial s_{\beta}}+\sum_{k}^{m+1 \ldots n} \gamma_{k i i} \frac{\partial \gamma_{k i i}}{\partial s_{e}}+\sum_{j}^{1 \ldots m} \gamma_{j \beta \beta} \gamma_{j e} \\
+\gamma_{\beta e e} \gamma_{\beta e}+2 \sum_{j}^{1 \ldots m} \gamma_{\beta j e} \gamma_{j \beta}+\sum_{k}^{m+1 \ldots n} \gamma_{k e \beta} \gamma_{k \beta}-B_{\beta} \gamma_{\beta e} \\
+\gamma_{e i i}\left[B_{\beta}^{2}-\frac{1}{2} \sum_{j}^{1 \ldots m} B_{j}^{2}+\frac{1}{2}\left(\gamma_{i \alpha, \alpha i}-\gamma_{i \beta, \beta i}-\gamma_{\alpha \beta, \beta a}\right)+\frac{1}{2} \sum_{k}^{m+1 \ldots n} \gamma_{k i i}^{2}\right]=0 .
\end{gathered}
$$

From the equation

$$
\gamma_{i \alpha, a i}=B_{q r, s t} \lambda_{i}^{q} \lambda_{\alpha}^{r} \lambda_{\alpha}^{s} \lambda_{i}^{t}
$$

we find

$$
\frac{\partial \gamma_{i \alpha, \alpha i}}{\partial s_{e}}=B_{g r, s t p} \lambda_{i}^{q} \lambda_{\alpha}^{r} \lambda_{\alpha}^{s} \lambda_{i}^{t} \lambda_{e}^{p}+2 \sum_{j}^{1 \ldots m}\left(\gamma_{j a} \gamma_{a j e}+\gamma_{j i} \gamma_{i j e}\right)
$$

$$
+2 \gamma_{a i, i e} \gamma_{a e e}+2 \gamma_{i a, a e} \gamma_{i e e} .
$$

In like manner we have

$$
\frac{\partial \gamma_{\beta i, i e}}{\partial s_{\beta}}=B_{q r, s t p} \lambda_{\beta}^{q} \lambda_{i}^{r} \lambda_{i}^{s} \lambda_{e}^{t} \lambda_{\beta}^{p}-\sum_{j}^{1} \varlimsup_{j i, i e}^{n} \gamma_{j \beta \beta}-\gamma_{i \beta, \beta e} \gamma_{i \beta \beta}
$$

$$
+\gamma_{\beta i, i \beta} \gamma_{e \beta \beta}+\sum_{k}^{m+1 \ldots n} \gamma_{\beta i, i k} \gamma_{e k \beta} .
$$

Hence making use of (20), we find

$$
\begin{aligned}
\frac{\partial \gamma_{\beta e}}{\partial s_{\beta}} & =\frac{\partial}{\partial s_{\beta}}\left(\gamma_{\beta i . i e}+\gamma_{\beta e e} \gamma_{e i i}\right)=B_{q r, s t p} \lambda_{\beta}^{q} \lambda_{i}^{r} \lambda_{i}^{s} \lambda_{e}^{t} \lambda_{\beta}^{p} \\
& -\sum_{j}^{1 \ldots m} \gamma_{j e} \gamma_{j \beta \beta}+\sum_{k}^{m+1 \ldots n}\left(\gamma_{\beta k} \gamma_{e k \beta}-\gamma_{k i, i e} \gamma_{k \beta \beta}\right)+\gamma_{\beta i, i \beta} \gamma_{e \beta \beta} \\
& +\gamma_{\beta e e} \gamma_{\beta i, i e}+\gamma_{e i i}\left(\gamma_{\beta e, e \beta}-\frac{\partial \gamma_{e i i}}{\partial s_{e}}-\gamma_{e i i}^{2}-\gamma_{\beta e e}^{2}-\sum_{k}^{m+1 \ldots n} \gamma_{k e e} \gamma_{k \beta \beta}\right) .
\end{aligned}
$$


Substituting in (72), we obtain

(76)

$$
\begin{aligned}
& -B_{\beta} \gamma_{\beta e}+\sum_{k}^{m+1 \ldots n} \gamma_{k i i}\left[-\gamma_{k i i} \gamma_{e i i}+\gamma_{e k i}\left(\gamma_{i k k}-\gamma_{i e e}\right)\right] \\
& +\gamma_{e i i}\left(\gamma_{\beta e, e \beta}-\gamma_{e i, i e}\right)+\gamma_{\beta i, i \beta} \gamma_{e i i}+\gamma_{e i i}\left[B_{\beta}^{2}-\frac{1}{2} \sum_{j}^{1} B_{j}^{2}+\frac{1}{2} \sum_{k}^{m+1 \ldots n} \gamma_{k i i}^{2}\right.
\end{aligned}
$$$$
\left.+\frac{1}{2}\left(\gamma_{i \alpha, \alpha i}-\gamma_{i \beta, \beta i}-\gamma_{\alpha \beta, \beta \alpha}\right)\right]+B_{q r, s t p}\left[\lambda_{\beta}^{q} \lambda_{i}^{r} \lambda_{i}^{s} \lambda_{e}^{t} \lambda_{\beta}^{p}\right.
$$$$
\left.+\frac{1}{2}\left(\lambda_{i}^{q} \lambda_{\alpha}^{r} \lambda_{\alpha}^{s} \lambda_{i}^{t}-\lambda_{i}^{q} \lambda_{\beta}^{r} \lambda_{\beta}^{s} \lambda_{i}^{t}-\lambda_{\alpha}^{q} \lambda_{\beta}^{r} \lambda_{\beta}^{s} \lambda_{\alpha}^{t}\right) \lambda_{e}^{p}\right]=0
$$

In consequence of (61) and (62) we have

$$
B_{q r, s t p} \lambda_{i}^{q} \lambda_{a}^{r} \lambda_{a}^{s} \lambda_{i}^{t} \lambda_{e}^{p}=B_{q r, s t p}\left(\lambda_{\alpha}^{q} \lambda_{i}^{r} \lambda_{i}^{s} \lambda_{e}^{t} \lambda_{\alpha}^{p}+\lambda_{i}^{q} \lambda_{\alpha}^{r} \lambda_{\alpha}^{s} \lambda_{e}^{t} \lambda_{i}^{p}\right)
$$

From (52) and (74) it follows that

$$
B_{q r, s t p}\left(\lambda_{\beta}^{q} \lambda_{i}^{r} \lambda_{i}^{s} \lambda_{e}^{t} \lambda_{\beta}^{p}-\lambda_{\beta}^{q} \lambda_{a}^{r} \lambda_{\alpha}^{s} \lambda_{e}^{t} \lambda_{\beta}^{p}\right)
$$

$$
=\sum_{k}^{m+1 \ldots n}\left(\gamma_{k i, i e}-\gamma_{k \alpha, \alpha e}\right) \gamma_{k \beta \beta}+\gamma_{e i i}\left(\gamma_{\beta \alpha, \alpha \beta}-\gamma_{\beta i, i \beta}\right) .
$$

From (20) it follows that

$$
\gamma_{k i, i e}-\gamma_{k \alpha, a e}=\gamma_{e k i}\left(\gamma_{i e e}-\gamma_{i k k}\right)-\gamma_{e k \alpha}\left(\gamma_{a e e}-\gamma_{\alpha k k}\right) \quad(e \neq k) .
$$

Substituting in (76) from (77), (78), (79) and similar expressions we have

$$
\gamma_{e i i}\left[B_{\beta}^{2}-\frac{1}{2} \sum_{j}^{1 \ldots m} B_{j}^{2}-\frac{1}{2}\left(\gamma_{i \alpha, \alpha i}-\gamma_{i \beta, \beta i}-\gamma_{\alpha \beta, \beta \alpha}\right)\right.
$$

$$
\left.-\frac{1}{2} \sum_{k}^{m+1 \ldots n} \gamma_{k i i}^{2}\right]-B_{\beta} \gamma_{\beta e}=0
$$


In arriving at this result we made use of the fact that $\sum_{k}^{m+1 \ldots n} \gamma_{k i i} \gamma_{e k \beta}\left(\gamma_{\beta e e}-\gamma_{\beta k k}\right)=0$. For if $e$ and $k$ belong to different roots of (2), then $\gamma_{e k \beta}=0$ and if to the same root the expression in parenthesis vanishes, in consequence of equations analogous to (37). Hence (76) reduces to (80).

When we express the consistency of (71) and

$$
\frac{\partial B_{\beta}}{\partial s_{\alpha}}=\sum_{j}^{1 \ldots m} B_{j} \gamma_{\beta j \alpha}+B_{\alpha} B_{\beta}-\gamma_{a \beta} \quad(\alpha=1, \ldots, m ; \alpha, \beta \neq),
$$

we obtain

$$
\begin{aligned}
& \frac{1}{2} \frac{\partial}{\partial s_{\alpha}}\left(\gamma_{i \alpha, \alpha i}-\gamma_{i \beta, \beta i}-\gamma_{\alpha \beta, \beta \alpha}\right)+\sum_{k}^{m+1 \ldots n} \gamma_{k i i} \frac{\partial \gamma_{k i i}}{\partial s_{\alpha}}+\frac{\partial \gamma_{a \beta}}{\partial s_{\beta}} \\
& \quad+\gamma_{o \beta \beta}\left(\gamma_{i \alpha, \alpha i}-\gamma_{i \beta, \beta i}\right)+\sum_{j \neq \alpha}^{1 \ldots m} \gamma_{j \beta \beta} \gamma_{j \alpha}+\sum_{j \neq \beta}^{1 \ldots m}\left(\gamma_{j \alpha \beta}-2 \gamma_{j \beta \alpha}\right) \gamma_{\beta j}=0 .
\end{aligned}
$$

Proceeding as in the case of (72), we obtain

$$
B_{q r, s t p}\left(\lambda_{i}^{q} \lambda_{\alpha}^{r} \lambda_{\alpha}^{s} \lambda_{i}^{t} \lambda_{\alpha}^{p}-\lambda_{i}^{q} \lambda_{\beta}^{r} \lambda_{\beta}^{s} \lambda_{i}^{t} \lambda_{\alpha}^{p}-\lambda_{\beta}^{q} \lambda_{\alpha}^{r} \lambda_{\alpha}^{s} \lambda_{\beta}^{t} \lambda_{\alpha}^{p}+2 \lambda_{\beta}^{q} \lambda_{i}^{r} \lambda_{i}^{s} \lambda_{\alpha}^{t} \lambda_{\beta}^{p}\right)=0
$$

In consequence of (61) and (62) we have

$$
B_{q r, s t p} \lambda_{i}^{q} \lambda_{\beta}^{r} \lambda_{\beta}^{s} \lambda_{i}^{t} \lambda_{\alpha}^{p}=B_{q r, s t p}\left(\lambda_{\beta}^{q} \lambda_{i}^{r} \lambda_{i}^{s} \lambda_{\alpha}^{t} \lambda_{\beta}^{p}+\lambda_{i}^{q} \lambda_{\beta}^{r} \lambda_{\beta}^{s} \lambda_{\alpha}^{t} \lambda_{i}^{p}\right)
$$

Hence the preceding equation becomes

$$
B_{q r, s t p}\left[\lambda_{i}^{q} \lambda_{\alpha}^{r} \lambda_{1 \ell}^{s} \lambda_{i}^{t} \lambda_{\alpha}^{p}-\lambda_{i}^{q} \lambda_{\beta}^{r} \lambda_{\beta}^{s} \lambda_{\alpha}^{t} \lambda_{i}^{p}\right.
$$

$$
\left.-\lambda_{\beta}^{q} \lambda_{\alpha}^{r} \lambda_{\alpha}^{s} \lambda_{\beta}^{t} \lambda_{\alpha}^{p}+\lambda_{\beta}^{q} \lambda_{i}^{r} \lambda_{i}^{s} \lambda_{\alpha}^{t} \lambda_{\beta}^{p}\right]=0
$$

8. Particular solutions of the problem. If the functions $\gamma_{e i i}=0$, for $i=1, \ldots, m ; e=m+1, \ldots, n$, that is, if

$$
\alpha_{r s / t} \lambda_{e}^{r} \lambda_{i}^{s} \lambda_{i}^{t}=0 \quad(i=1, \ldots, m ; e=m+1, \ldots, n),
$$


it follows from (67) that

$$
\gamma_{\alpha i, i e}=0 \quad(\alpha, i=1, \ldots, m ; e=m+1, \ldots, n)
$$

and from (53) that $\gamma_{\alpha e}=0$. Hence the conditions (66), (68) and (80) are satisfied identically. The conditions (49) may be written

$$
\boldsymbol{B}_{q r, s t} \lambda_{\beta}^{q} \lambda_{i}^{r} \lambda^{s} \lambda_{e}^{t}=0(\beta, i=1, \ldots, m ; \alpha, e=1, \ldots, n ; \beta, i, \alpha, e \neq) .
$$

In addition to (84) equations (52) for $m>3$ lead to conditions of the form

$$
\boldsymbol{B}_{q r, s t}\left(\lambda_{\beta}^{q} \lambda_{\alpha}^{r} \lambda_{\alpha}^{s} \lambda_{i}^{t}-\lambda_{\beta}^{q} \lambda_{j}^{r} \lambda_{j}^{s} \lambda_{i}^{t}\right)=0 \quad(\beta, \alpha, i, j=1, \ldots, m ; \beta, \alpha, i, j \neq) .
$$

In addition we have (70) and (82). Conversely, if equations (83) are satisfied, we have $\gamma_{\text {eii }}=0$ for $i=1, \ldots, m ; e=m+1, \ldots, n$. Hence:

When the solutions of (3) satisfy equations (29), (39), and in addition sets of equations of the forms (70), (82), (83), (85) and (86) for each multiple root of the third or higher order of equations (2), there exists a normal n-riple in the space.

Equations (50) and (51) are satisfied by $B_{\alpha}=0(\alpha=1, \ldots, m)$, if

$$
\gamma_{\beta e}=0 \quad(\beta=1, \ldots, m ; e=1, \ldots, n ; \beta, e \neq),
$$

$$
\gamma_{\alpha \beta, \beta \alpha}-\sum_{k}^{m+1 \ldots n} \gamma_{k i i}^{2}=0
$$

Conversely, it follows from (68) that if equations (87) are satisfied and $\gamma_{\text {eii }} \neq 0$ for any $e=m+1, \ldots, n$, then the only solutions of (50) and (51) are $B_{a}=0$.

From (24) and (27) we have

$$
\begin{aligned}
& \alpha_{r s / t} \lambda_{\beta}^{r} \lambda_{e}^{s} \lambda_{e}^{t}+\alpha_{r s}\left(\lambda_{e}^{r} \lambda_{e}^{s}-\lambda_{\beta}^{r} \lambda_{\beta}^{s}\right) \gamma_{\beta e e}=0, \\
& \alpha_{r s / t} \lambda_{e}^{r} \lambda_{i}^{s} \lambda_{i}^{t}+\alpha_{r s}\left(\lambda_{i}^{r} \lambda_{i}^{s}-\lambda_{e}^{r} \lambda_{e}^{s}\right) \gamma_{e i i}=0 .
\end{aligned}
$$

By means of equations of this form and (53) equations (87) are expressible in terms of $\alpha_{r s}$, its first covariant derivative, $\boldsymbol{B}_{q r, s t}$ and $\lambda$ 's. Hence: 
When the solutions of (3) satisfy (29), (39), (49) and equations of the form (87) for each multiple root of (2) of the third or higher order, there exists a normal $n$-uple in the space.

If $\gamma_{a b}=0, \gamma_{e i i} \neq 0$, for a particular $\alpha=1, \ldots, m$ and a particular $e=m+1, \ldots, n$, from (66) and (68), on the assumption that all of the $B$ 's are different from zero, it follows that we must have

$$
\begin{aligned}
& \gamma_{\alpha e}=0 \quad(\alpha=1, \ldots, m ; e=m+1, \ldots, n), \\
& B_{\alpha} B_{\beta}+\gamma_{\beta \alpha}=0 \quad(\alpha, \beta=1, \ldots, m ; \alpha, \beta \neq) .
\end{aligned}
$$

Operating on (89) with $\frac{\partial}{\partial s_{e}}$ for $e=m+1, \ldots, n$ and making use of (54), we obtain

$$
\gamma_{\beta a e}\left(B_{\alpha}^{2}-B_{\beta}^{2}\right)-\sum_{j \neq \alpha}^{1 \ldots \gamma_{a j}^{m}} \gamma_{\beta j e}-\sum_{j \neq \beta}^{1} \gamma_{\beta j}^{m} \gamma_{a j e}+\frac{\partial \gamma_{\alpha \beta}}{\partial s_{e}}=0
$$

From (57) we have also

$$
-\frac{\partial \gamma_{\alpha \beta}}{\partial s_{e}}+\sum_{j \neq a}^{1 \ldots m} \gamma_{a j} \gamma_{\beta j e}+\sum_{j \neq \beta}^{1 \ldots m} \gamma_{\beta j} \gamma_{\alpha j e}+\gamma_{\beta a e}\left(\gamma_{i \alpha, a i}-\gamma_{i \beta, \beta i}\right)-2 \gamma_{e i i} \gamma_{\beta \alpha}=0
$$

From these equations it follows that

$$
\gamma_{\beta a e}\left(B_{\alpha}^{2}-B_{\beta}^{2}+\gamma_{i \alpha, a i}-\gamma_{i \beta, \beta i}\right)-2 \gamma_{e i i} \gamma_{\beta \alpha}=0
$$

Subtracting from (80) the equation obtained by interchanging $\alpha$ and $\beta$ in this equation, we obtain

$$
\gamma_{e i i}\left(B_{\alpha}^{2}-B_{\beta}^{2}+\gamma_{i \alpha, \alpha i}-\gamma_{i \beta, \beta i}\right)=0
$$

From this equation and (90), it follows that $\gamma_{\beta \alpha}=0$, which is inconsistent with (89).

9. General solution. We consider finally the general case when (88) is not satisfied. If in conformity with (66) we put

$$
\gamma_{\beta e}=\gamma_{e i i} \sigma_{\beta} \quad(\beta, i=1, \ldots, m ; e=m+1, \ldots, n)
$$


equation (68) becomes

$$
\gamma_{\beta \alpha}-B_{\beta} \sigma_{\alpha}+B_{\beta} B_{\alpha}=0
$$

Interchange $\alpha$ and $\beta$ and subtract; then

$$
\boldsymbol{B}_{\alpha}=\boldsymbol{B}_{\boldsymbol{\beta}} \frac{\boldsymbol{\sigma}_{\boldsymbol{\alpha}}}{\boldsymbol{\sigma}_{\boldsymbol{\beta}}}
$$

and (92) may be replaced by

$$
B_{\beta}^{2}-\sigma_{\beta} B_{\beta}+\frac{\sigma_{\beta}}{\sigma_{\alpha}} \gamma_{\beta \alpha}=0
$$

From equations of this form it follows that $\gamma_{\beta \alpha} / \sigma_{\alpha}=\gamma_{\beta i} / \sigma_{i}$ and consequently

$$
\sigma_{i} \gamma_{\beta \alpha}=\sigma_{\alpha} \gamma_{\beta i}=\sigma_{\beta} \gamma_{\alpha i}
$$

Moreover, from (91) and (95) we obtain

(96) $\gamma_{\beta a} \gamma_{i e}=\gamma_{\beta i} \gamma_{\alpha e}=\gamma_{\alpha i} \gamma_{\beta e}(\alpha, \beta, i=1, \ldots, m ; e=m+1, \ldots, n)$.

Equation (80) is reducible by means of (91) and (94) to

$$
\sum_{j}^{1 \ldots m} B_{j}^{2}+\left(\gamma_{i \alpha, \alpha i}-\gamma_{i \beta, \beta i}-\gamma_{\alpha \beta, \beta \alpha}\right)+\sum_{k}^{m+1 \ldots n} \gamma_{k i i}^{2}+\frac{2 \gamma_{\beta e}}{\gamma_{\alpha e}} \gamma_{\alpha \beta}=0
$$

Interchanging $\alpha$ and $\beta$ in these equations and subtracting, we obtain

$$
\gamma_{i a, a i}-\gamma_{i \beta, \beta i}+\left(-\frac{\gamma_{\beta e}}{\gamma_{a e}}-\frac{\gamma_{\alpha e}}{\gamma_{\beta e}}\right) \gamma_{\alpha \beta}=0
$$

$$
(\alpha, \beta, i=1, \ldots, m ; e=m+1, \ldots, n) \text {. }
$$

Hence (97) reduces to

$$
\sum_{j}^{1 \ldots m} B_{j}^{2}-\gamma_{\alpha \beta, \beta a}+\left(\frac{\gamma_{\beta e}}{\gamma_{a e}}+\frac{\gamma_{\alpha e}}{\gamma_{\beta e}}\right) \gamma_{\alpha \beta}+\sum_{k}^{m+1 \ldots n} \gamma_{k i i}^{2}=0 .
$$


By means of equations of the form (94) this is reducible to

$$
\sum_{j}^{1 \ldots m} \gamma_{j e} B_{j}=\gamma_{e i i}\left[\gamma_{\alpha \beta, \beta \alpha}-\sum_{k}^{m+1 \ldots n} \gamma_{k i i}^{2}+\sum_{j \neq a, \beta}^{1 \ldots m} \frac{\gamma_{j e}}{\gamma_{\alpha e}} \gamma_{j \alpha}\right]
$$

and this in turn is reducible, by means of equations of the form (93), to

$$
B_{\alpha} \sum_{j}^{1} \gamma_{j e}^{2}=\gamma_{e i i}\left[\left(\gamma_{\alpha \beta, \beta \alpha}-\sum_{k}^{m+1} \gamma_{k i i}^{2}\right) \gamma_{\alpha e}+\sum_{j \neq \alpha, \beta}^{1 \ldots m} \gamma_{j e} \gamma_{j \alpha}\right] .
$$

As a consequence we must have

$$
\left(\gamma_{\alpha \beta, \beta \alpha}-\gamma_{\alpha i, i \alpha}\right) \gamma_{\alpha e}=\sum_{j \neq \alpha, i}^{1 \ldots m} \gamma_{j e} \gamma_{j \alpha}-\sum_{j \neq \alpha, \beta}^{1 \ldots m} \gamma_{j e} \gamma_{j \alpha}=\gamma_{\beta e} \gamma_{\beta \alpha}-\gamma_{i e} \gamma_{i \alpha}
$$

which because of (96) is equivalent to an equation of the form (98).

When the expressions for $B_{\alpha}(\alpha=1, \ldots, m)$ from (99) are substituted in (54), (71) and (94), we obtain the set of conditions to be satisfied in addition to those previously found, and thus the problem is reduced to an algebraic one. Recapitulating we have:

If the solutions of (3) satisfy (29), and for each multiple root equations of the form (39), (66), (70), (82), (85), (86), (96), (98) and the equations resulting from the substitution of the functions $B_{\alpha}$ given by (99) in (54), (71) and (94), the space adnits a normal n-uple.

From the manner in which these conditions were derived it is clear that while all of them are necessary, they are not necessarily independent. However, they are necessary and sufficient to determine whether the space possesses a normal $n$-uple.

Princeton University, PRinceton, N. J.

Note added in proof. In considering the particular solutions in $\S 8, I$ omitted the possibility of one or more of the $B$ 's being zero, but not all of them. For $B_{1}=0$, we have from (68) $\gamma_{e i i} \gamma_{\beta 1}-B_{\beta} \gamma_{1 e}=0(\beta=1, \ldots, m ; e=m+1, \ldots, n)$ and from (54) $\sum_{i}^{1 \ldots m} B_{i} \gamma_{1 i e}-\gamma_{1 e}=0(e=1, \ldots, n)$. When the expression for $B_{\beta}$ from the first is substituted in the second, we obtain one set of conditions, and when substituted in (51) and (54) the others to be added to (29), (39), (66), (70), (85) and (86). 